\title{
Study of Charm Fragmentation at H1
}

\author{
Juraj Bracinik for H1 Collaboration \\ School of Physics and Astronomy \\ University of Birmingham - UK
}

\begin{abstract}
The differential cross sections of $D^{* \pm}$ mesons as a function of two scaling variables $\mathrm{z}_{\mathrm{hem}}$ and $z_{j e t}$ are measured in DIS and are used to extract free parameters of nonperturbative fragmentation functions for QCD based models. When the presence of a $D^{* \pm}$ jet with $E_{T}^{*}>3 \mathrm{GeV}$ is required, the parameters extracted from the two distributions are in agreement, and agreement is also found with the value extracted from $e^{+} e^{-}$data by ALEPH. However, for the event sample without such a jet, significantly different parameters for the fragmentation functions are needed to describe the data.
\end{abstract}

\section{Introduction and definition of the experimental observables}

The inclusive cross section for the production of a heavy hadron in $e p$ collisions can be written as the convolution of three terms describing the structure of the proton, the hard scattering and the transition of partons into colourless hadrons respectively. The term describing the transition of partons to hadrons contains a non-perturbative component (in the following called the fragmentation function) which needs to be studied experimentally. Charm fragmentation has been studied extensively in $e^{+} e^{-}$colli-

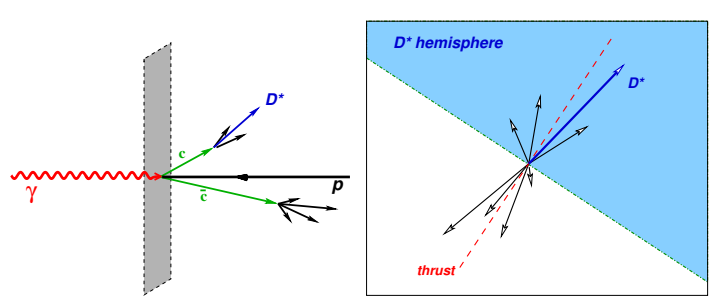

Figure 1: Kinematics of charm production in DIS as used for the definition of $\mathrm{z}_{\mathrm{hem}}$.

sions and several phenomenologic models have been developed to describe it. For example, in the model of independent fragmentation the charm quark picks up a light quark from the vacuum, forming a heavy hadron. The longitudinal momentum transfer from the parton to the hadron is described by a fragmentation function. In the case of the string model, hadrons are produced in the process of string breaking, and the fragmentation function describes the fraction of the string momentum transferred to the heavy hadron. Several functions are used to parametrise the fragmentation function of charm, for example by Peterson et al., with $D_{k}^{H}(z) \sim 1 / z[1-(1 / z)-\varepsilon(1-z)]^{2}$ or by Kartvelishvili et al. with $D_{k}^{H}(z) \sim z^{\alpha}(1-z)$, where $\varepsilon$ and $\alpha$ are free parameters.

A priori, it is not obvious that models developed to describe charm fragmentation in $e^{+} e^{-}$should work well also in the case of $e p$. In the case of $e^{+} e^{-}$, in the leading order the charm quarks are produced in a color singlet state, however in the case of $e p$ the charm and anticharm quarks are produced in a colour octet state. In addition, initial state gluon radiation is present in the case of $e p$ collisions. The difference of the production mechanism in $e^{+} e^{-}$and $e p$ influences the choice of the observable to study charm fragmentation. In $e^{+} e^{-}$the natural choice is to measure the differential cross section of a heavy hadron as a function of the scaled momentum $z=E_{H} / E_{\text {beam }}=E_{H} /(\sqrt{s} / 2)$. It is not possible to use the same observable in the case of $e p$. For the latter two different observables have been 

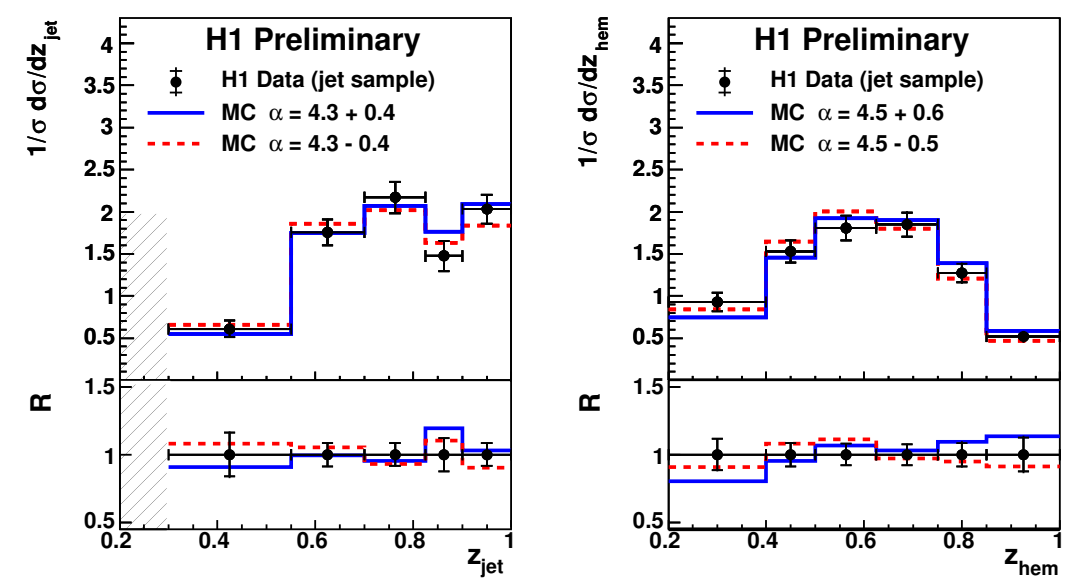

Figure 2: Normalised $D^{* \pm}$ meson cross sections as a function of $\mathrm{z}_{\mathrm{jet}}$ and $\mathrm{z}_{\mathrm{hem}}$. The data are compared with MC predictions of RAPGAP, using the Kartvelishvili parametrisation with the parameter $\alpha$ fitted to the data.

proposed instead. In the case of the so-called jet observable, the momentum of the charm quark is approximated by the momentum of the jet containing a $D^{* \pm}$ meson $\left(D^{* \pm}\right.$ jet $)$. The jet scaling variable is then defined as $\mathrm{z}_{\text {jet }}=\left(E^{*}+p_{L}^{*}\right)_{D^{* \pm}} /\left(E^{*}+p^{*}\right)_{j e t}$, where $E_{j e t}^{*}, p_{j e t}^{*}$ are the energy and the momentum of the $D^{* \pm}$ jet in the $\gamma^{*} p$ rest-frame, $E_{D^{* \pm}}^{*}$ is the energy of the $D^{* \pm}$ meson belonging to the jet, and $p_{L D^{* \pm}}^{*}$ is its momentum component parallel to the jet axis. In the case of the hemisphere observable, the kinematics of leading order charm production is taken into account. Looking at charm production in the $\gamma^{*} p$ rest-frame one can see that both quarks are boosted into the direction of the incoming photon (see Fig. 1 left), because while the photon interacts with its full energy, the gluon carries typically a small fraction of the proton momentum. Both $c$ and $\bar{c}$ are balanced in their $p_{T}$, allowing to divide the particles in the event into two groups, each of them containing mainly products of fragmentation of one of two quarks. The momenta of all particles in the photon hemisphere are projected onto the plane perpendicular to the $\gamma^{*} p$ axis, and the thrust axis in this plane is found. The line perpendicular to the thrust axis is used to divide the plane into two parts (see Fig. 1 right). The particles lying in the same part of the plane as the $D^{* \pm}$ meson are considered to be the products of the same quark, and the sum of their energy and momentum is used to approximate the energy and momentum of the original quark. The observable is then defined as $\mathrm{z}_{\mathrm{hem}}=\left(E^{*}+p_{L}^{*}\right)_{D^{* \pm}} / \sum_{h e m}\left(E^{*}+p^{*}\right)_{i}$. The two variables, $\mathrm{z}_{\mathrm{hem}}$ and $\mathrm{z}_{\mathrm{jet}}$, have different sensitivity to gluon radiation and details of the hadronization process, and the differential cross sections are expected to be different, however, a model providing a very good description of the underlying physics should be able to describe both variables using the same fragmentation function.

DIS 2008 

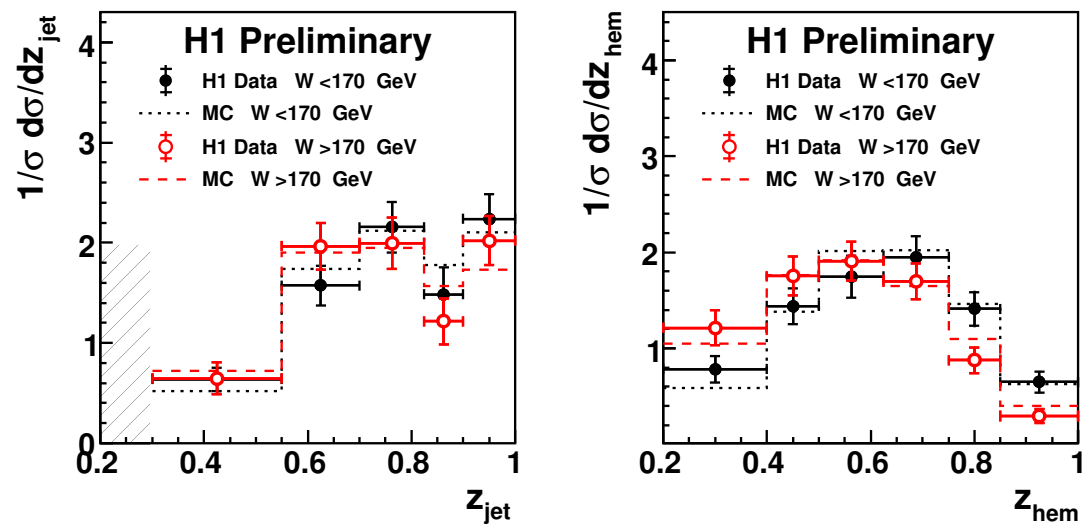

Figure 3: Normalised $D^{* \pm}$ meson cross sections as a function of $\mathrm{z}_{\text {hem }}$ and $\mathrm{z}_{\text {jet }}$ for $W<$ $170 \mathrm{GeV}$ and $W>170 \mathrm{GeV}$, (sample of events with a $D^{* \pm}$ jet in the event) with MC predictions of RAPGAP, and the fragmentation parameter $\alpha$ tuned to the full sample.

\section{Experimental results}

The normalised $D^{* \pm}$ meson cross sections as a function of $\mathrm{z}_{\mathrm{hem}}$ and $\mathrm{z}_{\mathrm{jet}}$ are shown in figure 2 . The measurement was performed by the H1 collaboration in DIS $\left(2<Q^{2}<100 \mathrm{GeV}^{2}\right.$ and $0.05<y<0.7)$ with the $D^{* \pm}$ meson fulfilling the conditions $\left|\eta_{D^{* \pm}}\right|<1.5$ and $1.5<$ $p_{T}\left(D^{* \pm}\right)<15 \mathrm{GeV}$. In addition, the presence of a $D^{* \pm}$ jet with $E_{T}^{*}\left(D^{* \pm} j e t\right)>3 \mathrm{GeV}$ in the $\gamma^{*} p$ rest-frame is required. The data are corrected for detector effects and QED radiation, and the $D^{* \pm}$ mesons due to B-hadron decays are subtracted. The sample used for this analysis corresponds to an integrated luminosity of $47 \mathrm{pb}^{-1}$. As expected, the two differential cross sections have a different shapes. The difference in the highest $z_{\text {jet }}$ bin being mainly due to a significant fraction of $D^{* \pm}$ jets consisting of a $D^{* \pm}$ meson only.

The data are used to fit the parameters of the Peterson and Kartvelishvili fragmentation functions for several QCD based models. The model predictions for various values of the free parameters $\varepsilon$ and $\alpha$ are compared to the data, and the best value is defined by the minimum of $\chi^{2}$. The shape of the $\chi^{2}$ distribution is used to determine its error. The fitted value for $\alpha$ or $\varepsilon$ depends on the setting used for other free parameters of the model. For example, in Fig. 2 one can see the data compared with the prediction of the LO+PS model RAPGAP, interfaced with the Lund string model as implemented in PYTHIA. Here, the PYTHIA parameter setting tuned by ALEPH, with a significant fraction of $D^{* \pm}$ mesons produced in decays of still heavier states, together with the Kartvelishvili fragmentation function are used. The data are well described by the model, and the parameters $\alpha$ fitted to the distributions of $z_{\text {hem }}$ and $z_{\text {jet }}$ separately are in good agrement with each other. The description of the data is good also for the Peterson parametrization, the extracted values $\varepsilon\left(z_{\text {hem }}\right)=0.029_{-0.005}^{+0.007}$ and $\varepsilon\left(z_{\text {jet }}\right)=0.035_{-0.006}^{+0.007}$ are in agreement with the value $\varepsilon=0.04$ obtained by the ALEPH collaboration. This result is consistent with the hypothesis of fragmentation universality between $e p$ and $e^{+} e^{-}$processes.

In order to study if the $Q^{2}$ and the $W$ dependence of the fragmentation observables can 
be described by the QCD models using the fragmentation parameters tuned to the full data sample, the $D^{* \pm}$ meson cross section as a function of $\mathrm{z}_{\mathrm{hem}}$ and $\mathrm{z}_{\text {jet }}$ are also measured in two bins in $Q^{2}\left(2<Q^{2}<10 \mathrm{GeV}^{2}\right.$ and $\left.10<Q^{2}<100 \mathrm{GeV}^{2}\right)$ and in $W(W<170 \mathrm{GeV}$ and $W \geq 170 \mathrm{GeV}$ ). As can be seen in Fig. 3, the data are well described by the QCD models. In the case of the massive $\mathrm{NLO}\left(\mathcal{O}\left(\alpha_{\mathrm{s}}^{2}\right)\right)$ calculation HVQDIS, the data are well described fitting the Kartvelishvili fragmentation function, while fitting the Peterson fragmentation function leads to a bad description.

The hemisphere observable allows to investigate the fragmentation of charm close to the kinematic threshold by selecting a sample of events fulfilling the nominal requirements on the DIS and $D^{* \pm}$ meson phase space, but requiring that there are no $D^{* \pm}$ jets with an $E_{T}^{*}>3 \mathrm{GeV}$ in an event.

The normalised $D^{* \pm}$ meson cross sections as a function of $\mathrm{z}_{\mathrm{hem}}$ for the sample without a $D^{* \pm}$ jet are compared to the prediction of RAPGAP with the Kartvelishvili fragmentation function and the value of $\alpha$ fitted to the nominal sample (dotted line) in Fig. 4. The distribution of the data is significantly harder then the prediction of the model. Fitting the value of $\alpha$ to the sample without a $D^{* \pm}$ jet leads to the value of $\alpha=10.3 \pm 1.5$, which significantly different from the value extracted using the nominal sample. The NLO calculation of HVQDIS

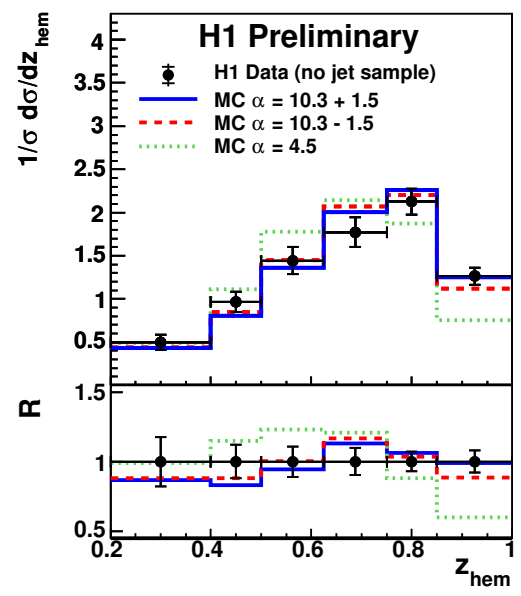

Figure 4: Normalised $D^{* \pm}$ meson cross sections as a function of $z_{h e m}$ for the sample of events requiring that there is no $D^{* \pm}$ jet in the event compared with MC predictions of RAPGAP using the ALEPH parameter setting with Kartvelishvili fragmentation function.

fails to describe the event sample without a

$D^{* \pm}$ jet $\left(\chi_{\min }^{2} \approx 40 / 4\right)$. The requirement of a significantly different fragmentation parameter for events close to the kinematic threshold can be considered as a signal of inadequacy of current QCD models and possibly of the simple parametrisations used for the fragmentation function.

\section{Acknowledgements}

I would like to thank Zuzana Rúriková and Günter Grindhammer for their impressive patience.

\section{Bibliography}

\section{References}

[1] Slides: http: //indico. cern. ch/contributionDisplay $\cdot$ py? contribId=236\&sessionId=14\&conf Id=24657 